\title{
EL ENFERMO TERMINAL Y LA EUTANASIA
}

Isa Fonnegra de Jaramillo*

\section{Conferencia pronunciada en el Hospital de San} José con motivo del centenario de la Sociedad de Cirugía de Bogotá, el día 19 de julio de 2002

Cuando la Sociedad de Cirugía de Bogotá se creó, hace 100 años, una conferencia sobre este tema jamás se habría dictado, sencillamente porque entonces ni el paciente terminal ni la eutanasia eran temas de discusión, dado que no representaban problema alguno.

Hoy me siento honrada y complacida por la invitación del doctor Tribín a abordar estos temas espinosos con ustedes y sinceramente espero que las ideas aquí expuestas sean recibidas a dos niveles diferentes: primero, a un nivel profesional ampliando quizás la óptica y el compromiso incancelable con nuestros pacientes en la fase terminal de su enfermedad. Y por otro lado, quizás más importante, a un nivel personal, abriendo un necesario espacio de cuestionamiento y reflexión acerca de nuestras posturas frente al morir humano y a la muerte propia, espacio que nos lleve a reexaminar viejas creencias tomando en cuenta los cambios tan radicales que la tecnología hoy disponible plantea en el manejo de una enfermedad y la nueva ética centrada en la autonomía y el respeto por los derechos individuales. No es posible que todavía muchos médicos continúen ejerciendo la medicina ciegamente aferrados a postulados que fueron válidos hace 20 o 50 años, cuando salimos de la universidad, pero que hoy necesitan ser replanteados en el ámbito personal, a la luz de lo nuevo y además, de la edad que hoy tenemos, de nuestras circunstancias vitales, de los principios y valores que hoy rigen nuestro acontecer, de las opciones disponibles en nuestro medio, y, lógicamente de la salud con que contamos.

Psicóloga Clínica y Tanatóloga. Fundadora y Presidente de Omega, una institución pionera en la atención paliativa a pacientes terminales, hoy dedicada a la asistencia en duelos. Es miembro del Consejo Directivo de la Fundación Pro Derecho a Morir Dignamente, del Instituto Colombiano de Estudios Bioéticos y del Grupo Internacional para el Estudio y el Trabajo con la Muerte, el Morir y el Duelo, IWG.
Muy seguramente la muerte que querríamos para nosotros hace 15 años, hoy no es muy posible y conviene dedicarle a este importante punto un tiempo que nos permita tomar decisiones sensatas y precisas sobre nuestro final, decisiones informadas.

Son estas consideraciones las que me movieron a aceptar el reto de compartir con ustedes esta mañana, sesenta minutos de reflexiones sobre los temas del morir y la muerte

\section{Sobre el morir de hoy}

Hoy en día se puede afirmar que la "muerte natural" como tal, no existe. La muerte que hace un siglo o menos, era una parte natural de la vida, con cuyo proceso y advenimiento aún los niños estaban familiarizados, y que tal como fue descrito por Philippe Aries, el moribundo presidía, es hoy ajena a muchos de nosotros. Presenciar la muerte y vivir con alguien su proceso de morir, son experiencias que gran porcentaje de la población, incluidos muchos estudiantes de medicina, reconoce no haber tenido.

Hemos alejado la muerte de nuestras vidas: hemos colocado en manos expertas pero ajenas su "manejo" y, adoptando pautas culturales vigentes en países con un alto nivel de desarrollo, pero igualmente con altos niveles de soledad y de carencia de vínculos familiares, hemos despojado a la muerte de su importancia, de los ritos y del luto que la hacían un evento relevante, $y$, por consiguiente, de su impacto emocional. Basta con observar a nuestro alrededor para confirmar estas observaciones: la muerte se ha medicalizado en exceso, alejándose del hogar, despojándose de los cuidados amorosos de los seres significativos y se ha institucionalizado, legislado y politizado. El escenario en que se produce la muerte hoy día es cualitativamente muy diferente de aquel que se daba el final de la vida a comienzos y aún, a mediados del siglo. 


\section{Sobre el médico y la muerte}

Muchos de los avances en la medicina y la tecnología contemporáneas, si bien han hecho posible eliminar plagas y enfermedades infecto-contagiosas, también han hecho posible el prolongar indefinidamente el proceso de VIVIR-MURIENDO de las largas y penosas enfermedades degenerativas, esas precisamente, que habrán de conducirnos a nosotros protagonistas del siglo XXI, muy seguramente, a una muerte lenta y terrible. La disponibilidad y en muchos casos el empleo indiscriminado y sin criterio, de sistemas artificiales de soporte vital en pacientes terminales, empañan y desvían la claridad en las decisiones médicas acerca de si se debe parar y cuándo, en qué momento, del recorrido de un ser humano hacia la muerte y poder así cambiar el objetivo de las intervenciones de CURAR a ALIVIAR. Si hemos tenido la experiencia de acercarnos al proceso de morir de un paciente o ser cercano, si hemos podido estar "al pie de su cama", nuestra perspectiva del morir humano será muy particular: no idealizada ni romantizada, sino realista, dura y conmovedora. En cualquier punto del camino que se recorre con una enfermedad incurable, se hace indispensable tomar decisiones; bien sea para revisar y replantear las previas o para formular nuevas expectativas, lo cual en la práctica se podría traducir crudamente, en permitir que sobrevenga la muerte ahora como parte natural del proceso de morir o en dilatarla más.

En Colombia al menos, la conclusión que me ha dejado la experiencia de cientos de casos en trance inminente de morir como consecuencia de su enfermedad, es que con muy contadas excepciones los médicos NO SABEN decir "Hasta aquí tiene sentido luchar" y plantear entonces el abstenerse de iniciar o el retirar las medidas artificiales de soporte vital. La formación universitaria les ha limitado su campo de acción y su misión profesional a CURAR, a vencer la muerte, no importa a qué costo físico, de sufrimiento, económico y familiar para el paciente. La muerte del enfermo es registrada por muchos como una derrota, un fracaso, porque su entrenamiento previo ha sido orientado hacia una meta: curar y salvar, con creencias muy arraigadas de que "mientras haya un soplo de vida, hay esperanza" pero ignorando el papel que especialmente al final de la vida, juega su CALIDAD y no la cantidad.
Dentro de los esquemas imperantes para la formación médica en Colombia, el tema del morir no es abordado; la importancia de la comunicación médico-paciente es subestimada y el proveer con herramientas y criterios éticos claros al joven médico para recomendar y tomar decisiones acertadas que respeten la autonomía del paciente terminal, es una tarea aún pendiente. Se espera que como arte de magia un médico pueda sortear exitosamente el evento del morir y de la muerte de su paciente, sin tener cómo apelar a patrones de experiencia previos, a esquemas de formación claros, a criterios definidos ni a ejemplos que le sirvan de referentes para poderse desempeñar con competencia y humanidad.

Ahora bien, en los años 70 surge una situación de conflicto. Con el advenimiento de la Tanatología con Kubler-Ross en Estados Unidos, con el establecimiento de la Medicina Paliativa en Inglaterra por Cicely Saunders y con la aparición simultánea en muchos países de los movimientos a favor del respecto al Derecho a Morir de un paciente, se configura un escenario de pugna entre dos fuertes tendencias: por un lado la incuestionada autoridad del médico ejercida tradicionalmente en forma paternalista y por otro la conciencia incrementada de la autonomía del paciente, esto es, del derecho a la autodeterminación en lo concerniente a la etapa final de la vida, explicitado en la enumeración creciente y reiterativa de los derechos del enfermo y en especial del derecho a rehusar tratamientos.

Desde hace más de 20 años me he comprometido activamente como lo han hecho mis colegas de las Fundaciones Omega y Pro Derecho a Morir Dignamente, con las tareas de difundir, asesorar y asistir directamente a los pacientes terminales y a sus familiares. Por ello mismo, he sido testigo de excepción de que en Colombia, de la teoría a la práctica hay un inmenso y a veces insalvable abismo: muchos médicos aceptan "intelectualmente" y a nivel oral la AUTONOMÍA del paciente, pero enfrentados al momento crítico de la toma de decisiones, la ignoran volviendo a imponer su sentir y su criterio, en la mayoría de los casos en detrimento de la calidad de vida del paciente terminal. Otros médicos, definitivamente no toman en cuenta la autodeterminación del paciente competente e informado y se aferran 
ciega e irrestrictamente a paradigmas vigentes hace décadas, según los cuales el médico “ordena” y el paciente coopera en forma pasiva e incuestionable.

Como en nuestro país no se trabaja aún dentro de la concepción de equipo multidisciplinario, la responsabilidad no se comparte y sólo le compete al médico, quien como se mencionó antes, con muy pocas excepciones, se guía por el principio de "salvar" la vida a todo costo, sin reconocer siquiera que existe un momento en el recorrido de la enfermedad en que con humildad y respeto por la dignidad humana, es necesario detenerse y suspender las medidas de soporte vital artificial como ventilación mecánica, diálisis, resucitación cardiopulmonar, etc., o abstenerse de iniciarlas si se consideran fútiles, innecesarias y generadoras de sufrimiento adicional para el enfermo y su familia, permitiendo así que sobrevenga una muerte inminente.

Ahora bien, no puedo dejar de mencionar aquí, que mi vida profesional se ha enriquecido con la presencia y cercanía de muchos médicos, humanos, responsables, prudentes y realistas en sus criterios y en su forma particularmente bella de relacionarse con sus pacientes, por quienes profeso admiración y de quienes he aprendido, manejando casos conjuntamente, el difícil y hermoso arte de acompañar a un buen morir.

\section{Acerca del enfermo terminal}

Se entiende por enfermo terminal al serhumano que sufre una enfermedad progresiva y deteriorante, para la cual ya han sido agotados todos los recursos disponibles y con una prospección de vida no mayor de seis meses.

El paciente terminal sufre: experimenta a diario micromuertes o micro-pérdidas de partes suyas, del futuro, de las expectativas, de roles previos, de la sensación de ser importante y útil a la sociedad, que preceden y anticipan su muerte final biológica. Esta situación genera en el enfermo reacciones naturales de ansiedad, angustia, de rabia, de depresión y duelo que lentamente, cuando le son permitidas por el medio, lo preparan para aceptar o resignarse a su muerte, pero que generalmente se ignoran, siendo atendido tan solo su dolor físico.

En muchos casos un paciente terminal desatendido médicamente, angustiado, aislado emocionalmente o en cuanto a sus necesidades de información y autodeterminación, o abandonado por su familia, manifiesta abiertamente su desesperanza y su deseo de morir, lo cual podría interpretarse como el requerimiento de eutanasia activa para poner fin a una vida de muy mala calidad. Pero, antes de pensar en concederle su deseo se hace imperativo revisar la eficacia médica de nuestro compromiso profesional con él.

Un paciente atendido en su dolor y en su sufrimiento, cuidado, querido y respetado seguramente no pide la eutanasia, porque en el fondo, querría vivir. Detrás del auge que tiene en la actualidad la literatura acerca del buen morir, de los casos como el Dr. KEVORKIAN, de los requerimientos de eutanasia activa o suicidio asistido, yace subyacente un reclamo por el CONTROL perdido, por la dignidad olvidada en medio de circunstancias degradantes que rodean la muerte de muchos en Colombia y en otros países del mundo.

La importancia de respetar y de incrementar el control del paciente, la autonomía y la calidad de vida no serán nunca sobreestimados. No todos los pacientes se sentirán tranquilos de permanecer en casa hasta su muerte: ésta es una opción más para explorar y además, habrá unos pocos casos en que la evolución y características de la enfermedad hagan indispensable la hospitalización. Pero, el facilitar los cuidados domiciliarios maximiza la independencia del paciente, permitiendo la participación activa de la familia en su cuidado y reduciendo sustancialmente la desorganización familiar que conlleva una hospitalización prolongada.

\section{Dilemas y situaciones de conflicto en la atención al paciente terminal}

Con el propósito de esquematizar esta presentación, voy a hacer una división arbitraria de seis áreas de posible conflicto en el manejo médico de la fase terminal de un enfermo. Ellas son:

- Manejo de la INFORMACIÓN.

- Manejo de la FAMILIA del paciente.

- Manejo del DOLOR y otros síntomas.

- Manejo del SUFRIMIENTO del paciente. 
- CALIDAD DE VIDA - CALIDAD DE MUERTE

\section{- PROCESO DE TOMA DE DECISIONES}

\section{Manejo de la informacíón}

Difíciles dilemas nos plantea el área de la información. Si partimos de la idea de que autonomía no es igual a arbitrariedad, deberemos informar al paciente en la medida en que él lo desee, tolere y necesite para poder contar con un consentimiento informado en lo referente al programa médico a seguir; información que presupone una comunicación abierta, directa y realista entre paciente, médico y familia. La brevedad de esta presentación no me permite adentrarme más en el apasionante tema del mito de la esperanza derrumbada por el conocimiento de la verdad, del manejo de niños y ancianos, etcétera.

\section{Manejo de la familia}

La enfermedad terminal no invade sólo al paciente sino en diferentes proporciones, a todos y cada uno de los miembros de esa familia. ¿Incluimos, entonces, a esa familia golpeada emocionalmente, desorganizada y angustiada en nuestras decisiones o la marginamos? ¿Nos reunimos periódicamente con todos para explicarles el curso de los acontecimientos y así facilitar su propio proceso de duelo anticipatorio, sin reforzar falsas expectativas? Recordemos que la muerte es un evento familiar; que de una u otra forma cada uno de los miembros se verá afectado por la enfermedad y la muerte de su ser querido y que en este proceso, tensiones preexistentes, recursos o debilidades particulares y reacciones individuales, suelen aparecer complicando el ya difícil panorama.

\section{Manejo dell dolor y de otros síntomas}

Maximizar la calidad de vida nos impone una guerra sin cuartel contra los síntomas que la deterioran. El esfuerzo médico debe ser incancelable en buscar los más eficientes mecanismos de control para el dolor y otros síntomas como disnea, fatiga, vómito, náusea, hipo, diarrea, etc., con el fin de otorgarle al pa- ciente la oportunidad de que, con su dolor controlado, pueda asumir y enfrentar su próxima muerte. Las clínicas de dolor y la generosidad en la prescripción de opiáceos, hacen que subsistan muy pocos e infortunados casos de pacientes que a pesar de los esfuerzos médicos, por la particular severidad de su enfermedad (por ejemplo cánceres infiltrantes, mesotelioma, poliartritis crónica, etc.), deban morir con dolor. Sin embargo, para estos casos se dispone de la sedación terminal estando amparada su aplicación en la ley del doble efecto.

Aceptar el principio del "doble efecto" en el cuidado del paciente terminal ha humanizado y mejorado la calidad de vida ante la muerte para muchos pacientes y además, absuelve al médico de la responsabilidad de haber acelerado la muerte cuando claramente es la intención aliviar los síntomas rebeldes del paciente. Esta flexibilidad también ha liberado al médico para poder emplear con seguridad y de acuerdo a la necesidad vigente en cada caso en particular, los medicamentos necesarios para tratar el dolor severo sin el temor a la adicción a los opioides o a que se le inculpe por abreviar el lapso de vida restante.

\section{Manejo del sufrimiento}

En la atención médica convencional, el sufrimiento de un paciente rara vez es contemplado y atendido adecuadamente. El cuerpo duele pero es el ser humano quien sufre, pero los esfuerzos médicos parecen minimizar su importancia, hasta el punto de ignorar el hecho de que con frecuencia una porción de sufrimiento es un efecto secundario del tratamiento orientado sólo a extender la vida restante a un paciente, así como también el temor del enfermo a que su caso ya no despierte interés en el médico, a "cansarlo" con sus quejas y por ende, a sentirse abandonado en su final, suele ser una de las más frecuentes causales de angustia en el paciente.

Explorar por qué sufre un paciente y buscar la ayuda necesaria para solucionarlo en lo posible, es una obligación moral de un médico tratante, sensible, humano y competente. 


\section{Calidad de vida - calidad de muerte}

Cada paciente es él más su historia y lo que para uno es calidad de vida para otro puede no serlo. El esfuerzo por la optimización de la calidad de la vida que queda no puede decaer hasta la muerte. Y así como hoy día una preocupación importante en la atención humanizada de pacientes es mantener la mejor calidad de vida posible, así mismo debe ser la lucha por la calidad de su morir. Procurarle todos aquellos "caprichos" que corresponden a sus necesidades, anticipar con la familia lo que se presume puede ser la causa desencadenante del evento de morir y reasegurarles que ofreceremos todas aquellas herramientas que eviten el pánico al final y que colaboren a manejar el caos y a permitir una muerte en circunstancias dignas, es hoy día el objetivo final de nuestra intervención con el paciente terminal. Tengamos presente que la muerte amable y digna de nuestro paciente debe ser vista por su médico como un logro meritorio y no como una derrota.

\section{Proceso de \\ toma de decisiones}

VIVIR es diferente a ESTAR VIVO y este criterio debe estar siempre presente a la hora de tomar las decisiones en el curso de la fase terminal de una enfermedad. El famoso argumento de "Mientras subsista un hilo de esperanza...o un soplo de vida", está sustentado en un sobredimensionamiento de la prolongación de la vida a cualquier costo. Indudablemente la tecnología actual tiene una poderosa capacidad de extender la vida, pero la misma intervención que puede prolongar el tiempo de vida, inintencionalmente puede contribuir a incrementar la desintegración, el sufrimiento y la indignidad al final.

Las decisiones deben ser constantemente examinadas y flexiblemente cambiadas de acuerdo a las necesidades diferentes que día a día plantea el manejo del paciente terminal y de su enfermedad particular. Lo que la semana pasada era válido es posible que hoy no lo sea. Y el proceso de tomarlas debe incluir un paciente suficientemente informado, o de no ser mentalmente competente, con una expresión previa, oral o escrita de sus preferencias. Ojalá exista un acuerdo entre paciente y médico que permita una responsabilidad compartida en un interjuego humano, considerado y respetuoso que tenga como base una comunicación abierta entre ambas partes.

Debemos tener presente que al final de nuestras vidas, el alivio del sufrimiento debe primar sobre la prolongación de la vida y que los principios éticos de autonomía, beneficencia - no maleficencia y justicia deben iluminar el sendero a seguir, sopesando siempre las decisiones a la luz de la ecuación costo-beneficio, sin perder de vista que hasta el final nuestra vida debe tener sentido, control y dignidad.

\section{Diferentes opciones para morir}

El auge de la autonomía del paciente y los crecientes avances tecnológicos de los que hoy dispone la medicina, dan pie en el siglo XXI a que cada ser humano disponga de diferentes alternativas para elegir, a la hora de pensar en el final de su preferencia. No sobra reiterar que tal determinación debe hacerse sustentada en una adecuada y clara información acerca de las opciones y de los pros y contras de cada una e idealmente debiera ser consultada y compartida con el médico personal.

Un necesario proceso de educación a la comunidad en estas materias; arrojaría resultados muy positivos con respecto a la sensatez y adecuación de las decisiones tomadas a las circunstancias particulares, tarea ésta que aún tiene un largo futuro por recorrer en nuestro medio, a pesar de los ingentes esfuerzos de entidades como DMD y Omega, dirigidos a concienciar a los médicos y a la población en general acerca de sus deberes y derechos en lo tocante al morir y la muerte. El documento "Esta es mi voluntad" es por excelencia el vehículo para consignar las preferencias personales y es gratuitamente distribuido por la Fundación Pro Derecho a Morir Dignamente.

\section{Muerte digna}

Es la muerte que muchos dicen que querrían tener. El paciente es tomado en cuenta por médicos y familiares como sujeto activo durante todo el proceso de la enfermedad previa. Quien muere sabe que está muriendo y está al tanto de lo que ocurre. 
Se trata entonces de la muerte OPORTUNA, sin dolor o con el menor dolor posible, con información adecuada, que ocurre preferiblemente en el ambiente familiar del paciente, en circunstancias que respetan sus deseos y que preservan su dignidad.

\section{Eutanasia pasiva}

Aunque este término ya no se usa, se entiende por ello el retirar oportunamente o el abstenerse de iniciar procedimientos o medidas de índole médica desproporcionadas en razón de reconocer que en el paciente terminal ya no son justificables. El respirador o ventilación mecánica, la diálisis, las cirugías mutilantes, los antibióticos y la sonda nasogástrica son algunos ejemplos de las medidas que deberían omitirse, tras evaluarlas como inútiles y tan solo prolongadoras de agonía.

Se deberá mantener sin embargo toda la medicación necesaria para aliviar el dolor, los síntomas o el sufrimiento, aún si su aplicación acortase la vida.

\section{Distanasia}

Es la muerte artificialmente dilatada mediante la utilización de medidas “terapéuticas” inútiles. El objetivo en este caso es la prolongación de la vida sin importar su calidad, gracias a esa aplicación indiscriminada de la tecnología médica disponible. Tales medidas extraordinarias, aunque fútiles, se emplean para diferir la muerte. El mantenimiento de un paciente terminal en una Unidad de Cuidados Intensivos es un clásico ejemplo de distanasia.

\section{Cuidados paliativos}

O “cuidados del bienestar" llamados así por tratarse de un programa interdisciplinario de asistencia integral a las necesidades físicas, emocionales, familiares y espirituales del paciente. El programa de cuidados paliativos aplicado a un paciente terminal está dirigido a CUIDAR y a ALIVIAR y ya no a CURAR lo que se sabe incurable, optimizando la calidad de vida restante.

Excluye por definición la aplicación de medidas de soporte artificial y los procedimientos ya inútiles en ese momento y acepta y permite la muerte sin dilatarla.

\section{Suicidio médicamente asistido}

En este caso, es el paciente mismo quien emplea o consume la sustancia que le ocasionará su muerte, pero es su médico quien se la ha prescrito o le ha facilitado obtenerla.

\section{Eutanasila activa}

Es la acción médica deliberada, generalmente en forma de inyección letal, que se aplica a un paciente con el fin explícito de terminar su vida. El paciente debe haberla solicitado consciente y expresamente y su caso debe ser terminal, irreversible, con dolor o sufrimiento insoportable e intratable.

Algo más sobre la eutanasia activa en Colombia como alternativa para morir

En 1997, a raíz de un fallo de la Corte Constitucional cuyo ponente fue el entonces Magistrado Carlos Gaviria Díaz, Colombia se convirtió en el primer país en tener en su sistema jurídico la autorización para practicar la eutanasia a enfermos terminales. Siguieron recientemente, Holanda, país donde sin ser legalizada se aplica desde hace muchos años y Bélgica. Australia y el Estado de Oregon en Estados Unidos han aprobado la práctica del suicidio médicamente asistido, no la eutanasia activa.

Los requerimientos vigentes exigidos para su aplicación son:

- Paciente con enfermedad incurable en fase terminal.

- Consciente y competente mentalmente.

- Que solicita expresamente a su médico, ayuda para poner fin a su vida debido a dolores insoportables e intratables.

No se incluyen ni el sufrimiento ni las enfermedades crónicas degenerativas. De tal manera, que si el paciente ejerce el derecho de solicitarla, que la Corte le ha reconocido, ajustándose a las condiciones estipuladas y si la solicitud se la hace a un médico, no habría motivo para que el médico se rehuse, exceptuando aquel caso en que el médico encuentre que satisfacer el deseo del paciente y practicarle la eutanasia entra en conflicto con sus principios éticos. Porque la misma autonomía que autoriza al paciente a solicitarla, autoriza al médico para negarse a practicarla. 
Desde entonces he tenido la impresión de que si bien para aquellos que conciben la vida como un bien valioso pero no divino y por tanto se permiten discrepar de la prohibición de la Iglesia Católica, este fallo representa una importante libertad de elegir; para muchos otros ha sido generador de pánico e inseguridad por considerar que podría llevar a abusos y a generalizaciones.

Desearía dejar en claro que tal y como está planteada en este momento, en Colombia, la eutanasia es una opción, despenalizada legalmente, o sea, permitida para todo aquel que libremente la elija como la mejor opción para morir. Pero no es una sugerencia ni una recomendación. Mucho menos, una obligación. Pecando de repetitiva, reitero que la misma autonomía que me autoriza a solicitarla, también me autoriza a cancelarla como alternativa viable para mí.

Ahora bien, un beneficio surge de toda esta polémica en torno a la eutanasia y a las otras formas de muerte asistida y es, que saca del closet de cada cual concepciones viejas, anquilosadas que no han sido sometidas a un re-examen. Sacude y cuestiona creencias preestablecidas y nos obliga a pensar y a comprometernos en la tarea de adueñarnos de nuestra propia muerte, de no delegarla, de asumirla con sus consecuencias cuando aún estamos lúcidos y mentalmente competentes. Así mismo, nos obliga a dar a conocer a médicos y familiares, nuestras personales decisiones en este cam- po, lo cual exonera al profesional de la salud de la agobiante tarea de tomar decisiones por otros y permite que dentro de lo humanamente posible, cada uno pueda aspirar a tener la muerte que quiere, ojalá en circunstancias respetables y respetuosas, dignas y humanas.

\section{Lecturas recomendadas}

Bejarano P, Jaramillo Isa. EDS Morir con Dignidad, Fundamentos del Cuidado Paliativo, Amazonas Editores, Bogotá, 1992.

Burnell GM. Final choices in an Age of Medical Technology, Plenum, New York. 1993.

Dunphy, Randall. Ethical Decision-Making. Palliative Care, European Journal Of Palliative Care, 1997, 4 (4): 126-128.

Jaramillo I. Cuidados Paliativos: Alternativas a la Eutanasia. El Tiempo, Lecturas Dominicales, Junio 1997.

Jaramillo I.De Cara a la Muerte. Editorial Andrés Bello, Chile. 2001.

Quill TE. Death and Dignity: Making Choices and Taking Charge. W.W. Norton, New York. 1993.

Quill TE. A Midwife through the Dying Process. Johns Hopkins University Press. 1996.

Saunders C. The Founding Philosophy Hospice: The Living Idea. London, Eduard Arnold 1981:4.

The right to choose to die en "THE ECONOMIST" Jun 21-27, 1997.

Saunders C, Kastembai, R. Hospice Care in the International Scene, Springer Publishing Co. New York. 1997.

Vachon M. Recent research into staff stress in palliative care, vol. 4:3 pp. 99-103.

Watters C. The benefits of home care for the terminally ill. European Journal of Palliative Care, Vol. 4:3 pp 96-98. 